Роль хрома в пищеварении у полигастричных животных (обзор)

\author{
О.В. Шочина, С.В. Лебедев, М.М. Поберухин \\ Федеральный научный центр биологических систем и агротехнологий Российской академии наук (2.Оренбург)
}

\begin{abstract}
Резюме. Хром $(\mathrm{Cr})$ - один из главных микроэлементов, участвующий в углеводном, белковом и липидном обменах. Добавление хрома в рационы улучшает иммунитет и повышает устойчивость животных к стрессу, что положительно сказывается на метаболизме энергии. Микроэлементу $\mathrm{Cr}$ принадлежит жизненно важная роль в действии инсулина, что проявляется в увеличении потребления глюкозы клетками и понижении концентрации свободных жирных кислот в крови. Он также необходим для хорошего функционирования $\beta$-клеток поджелудочной железы. Обычно хром пребывает в органических и неорганических формах с различной биологической доступностью и скоростью поглощения. Органические формы усваиваются значительно лучше неорганических за счёт более лёгкой адсорбции в кишечнике и отсутствию взаимодействия с другими компонентами корма. Актуальны исследования по включению в рацион животным микроэлементов в форме наночастиц. Данные биопрепараты представлены чаще всего в виде ультрадисперсных порошков металлов или их коллоидных растворов. Хром в форме ультрадисперсных частиц обладает пролонгированным действием в регуляции деятельности щитовидной железы, стабилизируя уровень глюкозы в крови, приводит к расщеплению излишнего жира и ускоряет липидный обмен, выводит из организма органические токсины, соли тяжёлых металлов. Наиболее оптимальный выбор органической формы хрома на данный момент - трёхвалентный в виде хромовых дрожжей. Неорганический хром неоднозначно влияет на физиологические и метаболические процессы в организме животных. Ключевые слова: жвачные животные, микроэлементы, пищеварение, обмен веществ, питательные вещества, органический хром, неорганический хром, поджелудочная железа.
\end{abstract}

UDC 636.085:577.17

\title{
The role of chromium in digestion in polygastric animals (review)
}

Oksana V Shoshina, Svyatoslav V Lebedev, Mikhail M Poberukhin

Federal Research Centre of Biological Systems and Agrotechnologies of the Russian Academy of Sciences (Orenburg, Russia)

\begin{abstract}
The addition of chromium to diets improves immunity and increases the resistance of animals to stress, which has a positive effect on energy metabolism. The microelement $\mathrm{Cr}$ plays a vital role in the action of insulin, which is manifested in an increase in glucose uptake by cells and a decrease in the concentration of free fatty acids in the blood. It is also essential for the good functioning of the $\beta$-cells of the pancreas. Chromium is usually found in organic and inorganic forms with varying bioavailability and absorption rates. Organic forms are absorbed much better than inorganic ones due to easier adsorption in the intestine and lack of interaction with other feed components. All organic forms of chromium have a positive effect on animal performance. Research on the inclusion of microelements in the form of nanoparticles in the diet of animals is relevant. These biological products are most often presented in the form of ultradispersed metal powders or their colloidal solutions. Chromium in the form of ultrafine particles activates metabolic processes in the body. It regulates the activity of the thyroid gland, stabilizes the level of glucose in the blood, leads to the breakdown of excess fat and accelerates lipid metabolism, removes organic toxins and salts of heavy metals from the body. The most optimal choice of the organic form of chromium at the moment is trivalent in the form of chromium yeast. The feed additive is a chromium preparation in its most physiologically accessible and biologically effective form, which is aimed at maximizing the beneficial effect on productivity, reducing morbidity and increasing the economic efficiency
\end{abstract}


of dairy farming. Inorganic chromium has a complex effect on the physiological and metabolic processes in the body of animals, while they are still poorly understood.

Keywords: ruminant, microelements, digestion, metabolism, nutrients, ruminants, organic chromium, inorganic chromium, pancreas.

\section{Введение.}

Микроэлемент хром играет главную роль в живом организме, это проявляется участием $\mathrm{Cr}$ в углеводном, белковом и липидном обменах. Добавки на основе хрома в рационы способствуют повышению иммунитета и устойчивости животных к стрессу, оказывая при этом хорошее влияние на метаболизм энергии (Фабер В. и др., 2020; Bompadre FV et al., 2020; Hasan HG et al., 2011).

Хром выполняет важную функцию в действии инсулина, увеличивая при этом потребление глюкозы клетками и понижая в крови концентрацию свободных жирных кислот. Особенно важно действие инсулина в периоды физиологических и технологических стрессов. Это служит поддержкой в эффективности метаболизма глюкозы, белка и жира (Шейда Е.В. и др., 2019; Лебедев С.В. и др., 2020; Lashkari S et al., 2018).

В клетках глюкоза трансформируется в энергию, которая используется в синтезе белка, росте безжировой (мышечной) ткани, поддержании жизнедеятельности клеток, а в итоге - в увеличении продуктивности животного (Козинец А.И. и др., 2020а).

Хром усиливает чувствительность к инсулину и поэтому может быть иммуномодулирующим. Значительное количество глюкозы используется в ответ на иммуногенный стимул. Дополнительный хром подавляет реакцию на инсулин и увеличивает циркулирующие нейтрофилы, но, не изменяет потребность иммунной системы в глюкозе после острой и интенсивной активации (Horst EA et al., 2018; Spears JW, 2019).

Также $\mathrm{Cr}$ необходим для хорошего функционирования $\beta$-клеток поджелудочной железы. Стресс и болезни оказывают отрицательное влияние на выработку инсулина поджелудочной железой. Это может послужить причиной ускорения метаболизма глюкозы и мобилизации хрома из депо организма, что в дальнейшем приведёт к увеличению экскреции хрома. Точное определение количества этого микроэлемента в организме животных достаточно сложно, ввиду того что метод измерения уровня хрома в крови малоэффективен (Budde AM et al., 2019; Chowdhury S et al., 2003).

Оптимальный уровень хрома оказывает положительное влияние на ход пищеварительных процессов, повышая переваримость сухого и органического вещества, сырого протеина, жира, клетчатки и БЭВ, использование азота, кальция, фосфора и хрома у жвачных животных. (Кокорев В.А. и др., 2017; Spears JW et al., 2017).

Экспериментальные исследования показали, что $\mathrm{Cr}$ может изменять производительность, иммунные реакции, метаболизм глюкозы и жирных кислот, а также антиоксидантный статус у молочных коров (Lashkari S et al., 2018).

Механизм биологического действия хрома и оптимальные концентрации содержания в рационах для правильного функционирования организма остаются до конца не изученными. Сr представляет собой связывающее вещество, этот факт объясняется в потенцировании действия инсулина (Stępniowska A et al, 2020). Низкомолекулярное Cr-связывающее вещество (LMWCr), активирующее фосфотирозин фосфатазу в изолированных мембранах адипоцитов, свидетельствует о функциональной связи между $\mathrm{Cr}$ и действием инсулина. Кинетические исследования показали, что LMWCr активирует активность фосфотирозин фосфатазы в мембранах адипоцитов, не имея собственной активности фосфатазы (Lukaski HC, 1999; Шейда Е.В. и др., 2018; Edwards KC et al, 2020).

Хром обычно пребывает в органических и неорганических формах с различной биологической доступностью и скоростью поглощения. Среди неорганических источников наиболее распространёнными формами хрома являются металлическая форма $(\mathrm{Cr} 0)$, трёхвалентная форма $(\mathrm{Cr} 3+)$ и шестивалентная форма (Cr6+) (Lu L et al., 2019). Именно трёхвалентный хром (Cr3+) обычно встречается в продуктах питания и является микронутриентом. Соли шестивалентного хрома (окись хрома, хромат) известны как соединения, высокотоксичные для человека и животных. Они 
не только обладают сильными канцерогенными и мутагенными свойствами, но, как известно, вызывают врождённые дефекты и снижают репродуктивное здоровье (Jobby R et al., 2018; Devoy J et al., 2019). Большая доза Cr (VI) может вызвать смерть животных и людей (Zou W et al., 2020).

В своём наиболее стабильном состоянии окисления $(\mathrm{Cr} 3+)$ хром осуществляет ряд важных функций для животных. Например, он вмешивается в метаболизм углеводов, белков и липидов, потенцирует действие инсулина как активного компонента фактора толерантности к глюкозе (GTF) и играет антистрессовую роль, снижая концентрацию кортизола в крови. Кроме того, он выполняет фундаментальную функцию в формировании и экспрессии генетической информации, оказывает ингибирующее действие на липогенную активность и улучшает всасывание аминокислоты в мышечных клетках для синтеза белка GTF, частично состоящего из молекул Сr, облегчает поступление глюкозы на клеточном уровне, повышая уровень инсулина в соматической клетке, и позволяет более широко использовать этот углевод, способствуя синтезу белка и, следовательно, снижению выработки жировой ткани (Haldar S et al., 2007; Vincent JB and Lukaski HC, 2018; Haq Z et al., 2016).

Большие дозы хрома, а в особенности шестивалентного вызывают отравления. Суточная норма хрома для животных составляет 300-500 мг/кг сухого вещества корма (Кононский А.И., 1992).

Часто в своих исследованиях учёные предлагают включать в рационы животных такие добавки хрома, в которых действующий микроэлемент находится в неорганической форме, в виду того что опыты на высокопродуктивных коровах доказали хорошее влияние органического хрома на воспроизводство подопытных животных (Ломаева А.А., 2018).

Из органических форм хрома наиболее выделяют $\mathrm{Cr}$ с метионином (CrMet), пиколинатом (CrPic), никотинатом (CrNic) и дрожжами (Cr-дрожжи). Органические формы хрома усваиваются значительно лучше неорганических за счёт более лёгкой адсорбции в кишечнике и отсутствия взаимодействия с другими компонентами корма. Все органические формы хрома оказывают положительное влияние на продуктивность животных (Han M et al., 2021).

Пахолкивым Н.И. с коллегами (2013) были проведены исследования по оценке влияния различных концентраций некоторых микроэлементов, в том числе органических и неорганических форм хрома, на жизнедеятельность микроорганизмов рубца крупного рогатого скота в условиях in vitro. По результатам исследований было выявлено, что включение хром-метионина в инкубационную среду искусственного рубца дало наиболее выраженный стимулирующий эффект на метаболическую активность микроорганизмов в сравнении с добавкой неорганического хрома. В результате было отмечено достоверное увеличение микробной массы, рост активности гидролитических ферментов микроорганизмов рубца, повышение целлюлозолитической и амилолитической активности бактерий рубца.

Хелат хрома метионина диссоциирует в пищеварительном тракте на два его компонента: хром в форме хрома (III) и метионин в форме DL. При всасывании хром связывается с транспортирующим железо белком плазмы, таким как трансферрин, и транспортируется в печень. Он также содержится в селезенке, почках, семенниках и костях. Отложение в мышцах происходит в очень изменчивой и низкой степени. Большая часть неабсорбированного хрома выводится с калом, моча является основным выделительным путём для поглощённого хрома как у животных, так и у людей: мочевой хром отражает потребление хрома с пищей, но он также содержится в молоке (Deng G et al., 2016; Vincent JB, 2019).

Хром DL-Met проявляет генотоксическую активность in vitro, которая не выражается in vivo после системного воздействия. Хотя данных о генотоксичности в месте контакта нет. Отмечено, что хром (III) не является канцерогенным и вряд ли представляет канцерогенный риск при уровнях, встречающихся в рационе питания (Bampidis V et al., 2020; Baggerman JO et al., 2020).

Биологическая роль пиколината хрома (III) состоит в том, что он участвует в утилизации глюкозы инсулином, предупреждает депрессию (Скорик Н.А. и др., 2020). Добавление 200 и 400 мкг 
хрома в качестве пиколината хрома может привести к цитотоксичности. Более высокий уровень добавок может также оказывать генотоксическое действие. Однако необходимы дальнейшие исследования, изучающие механизм действия пиколината хрома (Imamoğlu N et al., 2008). Добавки $\mathrm{CrPic}$ могут изменять углеводный обмен у первородящих коров в переходный период (Bompadre TFV et al., 2020; Williams CC et al., 2004).

Еще одним органическим соединением, используемым в рационах для откорма в условиях теплового стресса, является CrNic. Он содержит три единицы никотиновой кислоты на одну иона Cr. Эффекты, обнаруженные в исследованиях Toghyani с соавторами (2008) доказывают, что добавление $\mathrm{Cr}$ в виде CrNic в рацион оказывает положительное влияние на качество мяса и характеристики туши как в нормальных температурных условиях, так и в условиях теплового стресса. Эти исследования показали, что содержание белка в грудном мясе у животных, получавших CrNic, было выше по сравнению с этим же показателем у животных, получавших неорганический источник $\mathrm{Cr}$.

Большинство авторов предлагают хром в соединении с дрожжами как наиболее эффективную органическую добавку (Kani MM, 2015).

Добавление в рацион дойных коров дрожжей улучшало переваривание клетчатки и выход микробного азота, что объясняет улучшение удоев молока и молочных компонентов (Alhidary IA et al., 2018).

Актуальны исследования по включению в рацион животным микроэлементов в форме наночастиц. Данные биопрепараты представлены чаще всего в виде ультрадисперсных порошков металлов или их коллоидных растворов. Одна наиболее важная особенность наночастиц - способность в малых дозах активизировать физиологические и биохимические процессы. В результате этого значительно снижается количество используемого микроэлемента в составе рациона, что обеспечивает экономическую эффективность (Кравченко А.B., 2019; Untea AE et al., 2017; Lebedev S et al., 2018).

Хром в форме ультрадисперсных частиц активирует обменные процессы в организме. Он регулирует деятельность щитовидной железы, стабилизирует уровень глюкозы в крови, приводит к расщеплению излишнего жира и ускоряет липидный обмен, выводит из организма органические токсины, соли тяжёлых металлов (Шейда Е.В. и др., 2020а; 2020б; Титов В.Н. и др., 2016).

Замена классических источников микроэлементов хрома на органические и ультрадисперсные формы выражается в высокой удельной площади поверхности УдЧ, большей реакционной способностью и биодоступностью. В проведённом исследовании было показано, что наночастицы $\mathrm{Cr}_{2} \mathrm{O}_{3}$ в дозах от 50-100 мкг/кг корма влияют на формирование продуктивных качеств через стимулирование выработки пищеварительных ферментов у животных (Лебедев С.В. и др., 2019).

Включение в рационы опытных групп молодняка крупного рогатого скота наночастиц хрома в дозах 0,050 и 0,075 мг на 1 кг сухого вещества рациона увеличивает среднесуточные приросты на 6,6-3,3 \%, снижает себестоимость продукции на 4,9-1,4 \%. В итоге получают дополнительную прибыль в размере 4,9-18,0 рублей из расчёта на 1 голову (Козинец А.И. и др., 2020б).

В исследовании по оценке эффективности сухого вещества корма при добавлении наночастиц $\mathrm{SiO}_{2}$ и $\mathrm{Cr}_{2} \mathrm{O}_{3}$ действие $\mathrm{HЧ} \mathrm{Cr}_{2} \mathrm{O}_{3}$, наоборот, оказывает противоположный эффект на процессы переваримости. Было отмечено, что снижение концентрации нанопрепарата приводило к уменьшению показателя переваримости сухого вещества (при концентрации 0,5 М переваримость составила всего 49,4 \%). На основании этого использование данной наночастицы в качестве кормовой добавки неэффективно (Макаева А.М., 2017).

\section{Выводы.}

Таким образом, наиболее оптимальный выбор органической формы хрома на данный момент - трёхвалентный в виде хромовых дрожжей. Кормовая добавка представляет собой препарат хрома в его наиболее физиологически доступной и биологически эффективной форме, которая оказывает положительное влияние на продуктивность, снижение заболеваемости и повышение 
экономической эффективности молочного хозяйства. Неорганический хром сложно влияет на физиологические и метаболические процессы в организме животных, при этом они все ещё плохо изучены (Feng C et al., 2021; Кислякова Е.М. и Ломаева А.А., 2017).

Оптимальный уровень хрома в рационе положительно воздействует на процессы пищеварения, улучшая переваримость сухого вещества, сырого протеина, жира, клетчатки и БЭВ, а также повышая использование азота корма, кальция и фосфора. Также результаты исследований демонстрируют изменения в обмене минеральных веществ, что увеличивает отложения хрома в теле и выделения его из организма (Кокорев В.А. и др., 2015; Туаева Е.В. и Сковороднев Р.В., 2017).

Исследования выполнены в соответствии с планом НИР за 2021-2023 гг. ФГБНУ ФНЦ БСТ РАН (№ 0761-2019-0005)

\section{Литература}

1. Биологические эффекты, связанные с поступлением в организм цыплятбройлеров наночастиц хрома в разной дозировке / С.В. Лебедев, И.А. Гавриш, И.З. Губайдуллина, С.В. Шабунин // Сельскохозяйственная биология. 2019. Т. 54. № 4. С. 820-831. doi: 10.15389/agrobiology.2019.4.820rus [Lebedev SV, Gavrish IA, Gubajdullina IZ, Shabunin SV. Effects caused by different doses of dietary chromium nanoparticles fed to broiler chickens. Sel'skohozjajstvennaja biologija [Agricultural Biology]. 2019;54(4):820-831. (In Russ)]. doi: 10.15389/agrobiology.2019.4.820eng

2. Влияние органических и неорганических форм хрома на целлюлозолитическую и амилолитическую активность микроорганизмов рубца КРС / Н.И. Пахолкив, И.В. Лучка, Э.О. Дзень, Б.М. Куртяк, Б.О. Чорнушкин // Передгірне та гірське землеробство і тваринництво. 2013. № 55-1. C. 165-169. [Pakholkiv NI, Luchka IV, Dzen' EO, Kurtyak BM, Chornushkin BO. Vliyanie organicheskikh i neorganicheskikh form khroma na tsellyulozoliticheskuyu i amiloliticheskuyu aktivnost' mikroorganizmov rubtsa KRS. Peredgirne ta girs'kezemlerobstvo i tvarinnitstvo. 2013;55-1:165-169. (In Russ)].

3. Влияние различных форм хрома на обмен химических элементов в организме крыс линии Wistar / Е.В. Шейда, С.В. Лебедев, И.З. Губайдуллина, В.А. Рязанов, И.А. Гавриш // Известия Оренбургского государственного аграрного университета. 2019. № 2(76). C.167-171. [Sheida EV, Lebedev SV, Gubaidullina IZ, Ryazanov VA, Gavrish IA. Impact of different forms of chrome on the chemical elements metabolism in the organism of rats of the Wistar line. Izvestia Orenburg State Agrarian University. 2019;2(76):167-171. (In Russ)].

4. Влияние смеси незаменимых аминокислот в сочетании с кобальтом и хромом на химический состав и качество мяса телят казахской белоголовой породы / С.В. Лебедев, В.В. Гречкина, М.В. Клычкова, О.С. Мукашев // Животноводство и кормопроизводство. 2020. Т. 103. № 1. С. 168179. [Lebedev SV, Grechkina VV, Klychkova MV, Mukashev OS. The effect of a mixture of essential amino acids in combination with cobalt and chromium on the chemical composition and quality of beef of the Kazakh white-headed calves. Animal Husbandry and Fodder Production. 2020;103(1):168-179. (In Russ)]. doi: 10.33284/2658-3135-103-1-168

5. Влияние хрома на обмен веществ и молочную продуктивность коров / В.А. Кокорев, А.Б. Межевов, Н.И. Гибалкина, А.Н. Федаев, А.М. Гурьянов // Животноводство и ветеринарная медицина. 2015. № 3. С. 3-14. [Kokorev VA, Mezhevov AB, Gibalkina NI, Fedaev AN, Gur'yanov AM. Vliyanie khroma na obmen veshchestv i molochnuyu produktivnost' korov. Animal Agriculture and Veterinary Medicine. 2015;3:3-14. (In Russ)].

6. Изменение активности пищеварительных ферментов панкреатического сока под влиянием ультрадисперсных частиц $\mathrm{Cr}_{2} \mathrm{O}_{3}$ на фоне скармливания белковых рационов при выращивании крупного рогатого скота / Е.В. Шейда, С.В. Лебедев, С.А. Мирошников, В.В. Гречкина, В.А. Рязанов, О.В. Шошина // Животноводство и кормопроизводство. 2020а. Т. 103. № 4. С. 26-36. [Sheida EV, Lebedev SV, Miroshnikov SA, Grechkina VV, Ryazanov VA, Shoshina OV. Changes in the activity of 
digestive enzymes of pancreatic juice under the influence of ultrafine particles of $\mathrm{Cr} 2 \mathrm{O} 3$ against the background of feeding with protein diets raising cattle. Animal Husbandry and Fodder Production. 2020a;103(4):26-36. (In Russ)]. doi: 10.33284/2658-3135-103-4-26

7. Кислякова Е.М., Ломаева А.А. Влияние добавок органического хрома на продуктивные и репродуктивные показатели коров черно-пестрой породы // Ученые записки Казанской государственной академии ветеринарной медицины им. Н.Э. Баумана. 2017. Т. 232. № 4. С. 76-80. [Kislyakova EM, Lomaeva AA. Influence of organic chrome additives on productive and reproductive indicators of black and pestored breeds. Scientific Notes Kazan Bauman State Academy of Veterinary Medicine. 2017;232(4):76-80. (In Russ)].

8. Кононский А.И. Биохимия животных. 3-е изд., перераб. и доп. М.: Колос, 1992. 526 с. [Kononskii AI. Biokhimiya zhivotnykh. 3-e izd., pererab. i dop. Moscow: Kolos; 1992;526 p. (In Russ)].

9. Кравченко А.В. Влияние наночастиц хрома на качество туш, мяса и подкожного жира свиней // Зоотехническая наука Беларуси. 2019. Т. 54. № 2. С. 26-37. [Kravchenko AV. Effect of chromium nanoparticles on quality of carcass, meat and basing fat of pigs. Zootekhnicheskaya nauka Belarusi. 2019;54(2):26-37. (In Russ)].

10. Ломаева А.А. Показатели продуктивности коров чёрно-пёстрой породы при использовании в рационах органического хрома: дис. ... канд. с.-х. наук. Ижевск, 2018. 121 с. [Lomaeva AA. Pokazateli produktivnosti korov cherno-pestroi porody pri ispol'zovanii $\mathrm{v}$ ratsionakh organicheskogo khroma. [dissertation] Izhevsk; 2018:121 p. (In Russ)].

11. Олеиновые триглицериды пальмового масла и пальмитиновые триглицериды сливочного жира. Реакция пальмитирования, пальмитат калия, магния, всасывание энтероцитами жирных кислот и микробиота толстого кишечника / В.Н. Титов, А.В. Ариповский, В.В. Щекотов, А.П. Щекотова, В.В. Кухарчук // Клиническая лабораторная диагностика. 2016. Т. 61. № 8. С. 452-461. [Titov VN, Aripovskii AV, Shchekotov VV, Shchekotova AP, Kukharchuk VV. The oleic triglycerides of palm oil and palmitic triglycerides of creamy fat. The reaction of palmitoylation, potassium and magnesium palmitate, absorption of fatty acids by enterocytes and microbiota of large intestine. The Russian Clinical Laboratory Diagnostics Journal. 2016;61(8):452-461. (In Russ)]. doi: 10.18821/0869-2084-2016-61-8-452-461

12. Оценка влияния ультрадисперсных частиц $\mathrm{Cr}_{2} \mathrm{O}_{3}$ на метаболические процессы в организме телят, выращиваемых на белковых рационах / Е.В. Шейда, С.В. Лебедев, С.А. Мирошников, В.В. Гречкина, В.А. Рязанов // Животноводство и кормопроизводство. 2020б. Т. 103 № 4. С.14-25. [Sheida EV, Lebedev SV, Miroshnikov SA, Grechkina VV, Ryazanov VA. Assessment of influence of ultrafine particles of $\mathrm{CR}_{2} \mathrm{O}_{3}$ on metabolic processes in the body of calves raised on protein diets. Animal Husbandry and Fodder Production. 20206;103(4):14-25. (In Russ)]. doi: 10.33284/2658-3135-103-4-14

13. Применение наночастиц хрома в рационах молодняка крупного рогатого скота / А.И. Козинец, Т.Г. Козинец, О.Г. Голушко, М.А. Надаринская, М.С. Гринь, А.В. Соловьев // Научное обеспечение животноводства Сибири: материалы IV Междунар. науч.-практ. конф., (г. Красноярск, 14-15 мая 2020 года) / сост. Л.В. Ефимова, Ю.Г. Любимова. Красноярск: КрасНИИЖ ФИЦ КНЦ СО РАН, 2020a. C. 257-260. [Kozinets AI, Kozinets TG, Golushko OG, Nadarinskaya MA, Grin' MS, Solov'ev AV. Primenenie nanochastits khroma $\mathrm{v}$ ratsionakh molodnyaka krupnogo rogatogo skota. (Conference proceedings) Nauchnoe obespechenie zhivotnovodstva Sibiri: materialy IV Mezhdunar. nauch.-prakt. konf., (g. Krasnoyarsk, 14-15 maya 2020 goda). sost. Efimova LV, Lyubimova YuG. Krasnoyarsk: KrasNIIZh FITs KNTs SO RAN; 2020a: 257-260. (In Russ)].

14. Продуктивность полновозрастных коров при разных уровнях хрома в их рационах / В.А. Кокорев, Е.В. Болотин, Н.И. Гибалкина, Н.И. Федаев, А.М. Гурьянов // Животноводство и ветеринарная медицина. 2017. № 2(25). С. 20-30. [Kokorev VA, Bolotin EV, Rybalkin NI, Fedaev AN, Gur'yanov AM. Productivity of mature cows at different levels of chromium in their diets. Animal Agriculture and Veterinary Medicine. 2017;2(25):20-30. (In Russ)].

15. Продуктивность телят до 75-дневного возраста при использовании наночастиц хрома / А.И. Козинец, О.Г. Голушко, Т.Г. Козинец, М.А. Надаринская, М.С. Гринь, С.А. Гонакова, А.В. Соловьев // Актуальные проблемы интенсивного развития животноводства. 2020б. № 23-1. С. 128- 
135. [Kozinets AI, Golushko OG, Kozinets TG, Nadarinskaya MA, Grin' MS, Gonakova SA, Solov'ev AV. Produktivnost' telyat do 75-dnevnogo vozrasta pri ispol'zovanii nanochastits khroma. Aktual'nye problemy intensivnogo razvitiya zhivotnovodstva. 20206;23-1:128-135. (In Russ)].

16. Скорик Н.А., Алимов Р.P. Соединения хрома (III) с некоторыми органическими лигандами // Журнал неорганической химии. 2020. Т. 65. № 1. С. 16-24. [Skorik NA, Alimov RR. Chromium(III) compounds with some organic ligands. Russian Journal of Inorganic Chemistry. 2020;65(1):1624. (In Russ)]. doi: 10.31857/S0044457X2001016X

17. Сравнительная оценка эффективности переваривания кормов при внесении наночастиц металлов в условиях in vitro / А.Н. Макаева, К.Н. Атландерова, С.А. Мирошников, Д.Б. Косян // Известия Оренбургского государственного аграрного университета. 2017. №6(68). С. 178-180. [Makaeva AN, Atlanderova KN, Miroshnikov SA, Kosyan DB. Comparative evaluation of feeds digestion effectiveness as result of metal nanoparticles introduction under in vitro conditions. Izvestia Orenburg State Agrarian University. 2017;6(68):178-180. (In Russ)].

18. Туаева Е.В., Сковороднев Р.В. Влияние скармливания хрома в минеральной и органической форме молодняку крупного рогатого скота на их рост, развитие и обмен веществ // Агропромышленный комплекс: проблемы и перспективы развития: материалы междунар. науч.-практ. конф., посвященной Году экологии в России (г. Благовещенск, 5 апр. 2017 г.). Благовещенск: Дальневосточный ГАУ, 2017. Ч. 1. С. 177-180. [Tuaeva EV, Skovorodnev RV. Effect of feeding chromium mineral and organic form to young cattle in their growth, development and metabolism. (Conference proceedings) Agropromyshlennyi kompleks: problemy i perspektivy razvitiya: materialy mezhdunar. nauch.-prakt. konf., posvyashch. godu ekologii v Rossii. (g. Blagoveshchensk, 5 apr. 2017 g.). Blagoveshchensk: Dal'nevostochnyi GAU. 2017;1:177-180. (In Russ)].

19. Фабер В., Акмалиев Т., Гусева О. Минеральное питание жвачных // Животноводство России. 2020. № 5. С. 30-33. [Faber W, Akmaliev T, Guseva O. Mineral nutrition for ruminants. Zhivotnovodstvo Rossii. 2020;5:30-33. (In Russ)].

20. Хром, его роль в питании животных / Е.В. Шейда, С.В. Лебедев, И.А. Гавриш, Э.3. Губайдуллина // Мясное скотоводство - приоритеты и перспективы развития: материалы Междунар. науч.-практ. конф. (г. Оренбург, 25-26 апр. 2018 г.) / под общ. ред. чл.-корр. РАН С.А. Мирошникова. Оренбург: ФНЦ БСТ РАН, 2018. С. 165-167. [Sheida EV, Lebedev SV, Gavrish IA, Gubaidullina EZ. Khrom, ego rol' v pitanii zhivotnykh. (Conference proceedigs) Myasnoe skotovodstvo - prioritety i perspektivy razvitiya: materialy Mezhdunar. nauch.-prakt. konf. (g. Orenburg, 25-26 apr. 2018 g.). pod obshch. red. chl.-korr. RAN Miroshnikova SA. Orenburg: FNTs BST RAN; 2018:165-168. (In Russ)].

21. Alhidary IA, Alsofi MA, Abdoun KA, Samara EM, Okab AB, Al-Haidary AA. Influence of dietary chromium yeast supplementation on apparent trace elements metabolism in growing camel (Camelus dromedarius) reared under hot summer conditions. Trop Anim Health Prod. 2018;50(3):519524. doi: 10.1007/s11250-017-1462-x

22. Baggerman JO, Smith ZK, Thompson AJ, Kim J, Hergenreder JE, Rounds W, Johnson BJ. Chromium propionate supplementation alters animal growth performance, carcass characteristics, and skeletal muscle properties in feedlot steers. Transl Anim Sci. 2020;4(3):txaa146. doi: 10.1093/tas/txaa146

23. Bampidis V, Azimonti G, Bastos ML, Christensen H, Dusemund B, Durjava MK, Kouba M, Lopez-Alonso M, Puente SL, Marcon F, Mayo B, Pechova A, Petkova M, Ramos F, Sanz Y, Villa RE, Woutersen R, Gropp J, Mantovani A, Lopez-Galvez G. Safety and efficacy of Availa ${ }^{\circledR} \mathrm{Cr}$ (chromium chelate of DL-methionine) as a feed additive for dairy cows. EFSA J. 2020;18(2):e06026. doi: 10.2903/j.efsa.2020.6026

24. Bompadre TFV, Moretti DB, Sakita GZ, Ieda EH, Martinez MIV, Fernandes EAN, Machado-Neto R, Abdalla AL, Louvandini H. Long-term chromium picolinate supplementation improves colostrum profile of Santa Ines ewe. Biological Trace Element Research. 2020; 193(2):414-421. doi: 10.1007/s12011-019-01741-3

25. Budde AM, Sellins K, Lloyd KE, Wagner JJ, Heldt JS, Spears JW, Engle TE. Effect of zinc source and concentration and chromium supplementation on performance and carcass characteristics in feedlot steers. J Anim Sci. 2019;97(3):1286-1295. doi: 10.1093/jas/skz016 
26. Chowdhury S, Pandit K, Roychowdury P, Bhattacharya B. Role of chromium in human metabolism, with special reference to type 2 diabetes. Journal of the Association of Physicians of India. 2003;51:701-705.

27. Deng G, Dyroff SL, Lockart M, Bowman MK, Vincent JB. The effects of the glycation of transferrin on chromium binding and the transport and distribution of chromium in vivo. J Inorg Biochem. 2016;164:26-33. doi: 10.1016/j.jinorgbio.2016.08.008

28. Devoy J, Cosnier F, Bonfanti E, Antoine G, Nunge H, Lambert-Xolin A-M, Decret M-J, Douteau L, Lorcin M, Sebillaud S, Grossmann S, Michaux S, Müller S, Viton S, Seidel C, Gate L. Intraerythrocyte chromium as an indicator of exposure to hexavalent chromium: An in vivo evaluation in intravenous administered rat. Toxicol Lett. 2019;314:133-141. doi: 10.1016/j.toxlet.2019.07.020

29. Edwards KC, KimH, Vincent JB. Release of trivalent chromium from serum transferrin is sufficiently rapid to be physiologically relevant. J Inorg Biochem. 2020;202:110901. doi: 10.1016/j.jinorgbio.2019.110901

30. Feng C, Lin H, Li J, Xie B. Effects of dietary inorganic chromium supplementation on broiler growth performance: a meta-analysis. Peer J. 2021;9: e11097. doi: 10.7717/peerj.11097

31. Haldar S, Samanta S, Banarjee R, Sharma B, Ghosh TK. Glucose tolerance and serum concentrations of hormones and metabolites in goats (Capra hircus) fed diets supplemented with inorganic and organic chromium salts. Animal. 2007;1(3):347-356. doi: 10.1017/S1751731107393026

32. Han M, et al. Effects of organic chromium sources on growth performance, lipid metabolism, antioxidant status, breast amino acid and fatty acid profiles in broilers. J Sci Food Agric. 2021;101(9):3917-3926. doi: 10.1002/jsfa.11053

33. Haq Z, Jain RK, Khan N, Dar MY, Ali S, Gupta M, Varun TK. Recent advances in role of chromium and its antioxidant combinations in poultry nutrition: a review. Veterinary World. 2016;9(12):1392-1399. doi:10.14202/vetworld.2016.1392-1399

34. Hasan HG, Mahmood TJ, Ismael PA. Studies on the relationship between chromium (III) ion and thyroid peroxidase activity in sera of patients with thyroid dysfunction. Ibn AL-Haitham Journal for Pure and Applied Science. 2011;24(2):120-127.

35. Horst EA, Kvidera SK, Mayorga EJ, Shouse CS, Al-Qaisi M, Dickson MJ, Ydstie J, et al. Effect of chromium on bioenergetics and leukocyte dynamics following immunoactivation in lactating Holstein cows. Journal of Dairy Science. 2018;101(6):5515-5530. doi: 10.3168/jds.2017-13899

36. Imamoglu N, Uyanik F, Kocaoğlu GB, Erdem O, Cem LB, Dönmez Altuntaş H. Effects of chromium picolinate on micronucleus frequency and morphology of lymphocytes in calves. Biol Trace Elem Res. 2008;125(2):133-140. doi: 10.1007/s12011-008-8163-2

37. Jobby R, Jha P, Yadav AK, Desai N. Biosorption and biotransformation of hexavalent chromium [Cr(VI)]: A comprehensive review. Chemosphere. 2018;207:255266. doi: 10.1016/j.chemosphere.2018.05.050

38. Kani MM. The effects of different sources of organic and inorganic chromium on blood parameters of broiler chickens. Indian Journal of Science and Technology. 2015;8(28):1 7. doi: 10.17485/ijst/2015/v8i28/82778

39. Lashkari S, Habibian M, Jensen SK. A review on the role of chromium supplementation in ruminant nutrition-effects on productive performance, blood metabolites, antioxidant status, and immunocompetence. Biol Trace Elem Res. 2018;186(2):305-321. doi: 10.1007/s12011-018-1310-5

40. Lebedev S, Gavrish I, Rusakova E, Kvan O, Gubaidullina I. Influence of various chromium compounds on physiological, morpho-biochemical parameters, and digestive enzymes activity in Wistar rats. Trace Elements and Electrolytes. 2018; 35:242-245.

41. Lu L, Zhao LL, Dong SY, Liao XD, Dong XY, Zhang LY, Luo XG. Dietary supplementation of organic or inorganic chromium modulates the immune responses of broilers vaccinated with Avian Influenza virus vaccine. Animal. 2019;13(5):983-991. doi: 10.1017/S1751731118002379

42. Lukaski HC. Chromium as a supplement. Annu Rev Nutr. 1999;19:279-302. doi: 10.1146/annurev.nutr.19.1.279 
43. Spears JW, Lloyd KE, Krafka K. Chromium concentrations in ruminant feed ingredients. J Dairy Sci. 2017;100(5):3584-3590. doi: 10.3168/jds.2016-12153

44. Spears JW. Boron, chromium, manganese, and nickel in agricultural animal production. Biol Trace Elem Res. 2019;188(1):35-44. doi: 10.1007/s12011-018-1529-1

45. Stępniowska A, Tutaj K, Drażbo A, Kozłowski K, Ognik K, Jankowski J. Estimated intestinal absorption of phosphorus and its deposition in chosen tissues, bones and feathers of chickens receiving chromium picolinate or chromium nanoparticles in diet. PLoS One. 2020;15(11):e0242820. doi: 10.1371/journal.pone.0242820

46. Toghyani M, Khodami A, Gheisari AA. Effect of organic and inorganic chromium supplementation on meat quality of heat-stressed broiler chicks. American Journal of Animal and Veterinary Sciences. 2008;3(2):62-67. doi: 10.3844/ajavsp.2008.62.67

47. Untea AE, Varzaru I, Panaite TD, Habeanu M, Ropota M, Olteanu M, Cornescu GM. Effects of chromium supplementation on growth, nutrient digestibility and meat quality of growing pigs. South Afr J Anim Sci. 2017;47(3):332-341. doi: 10.4314/sajas.v47i3.10

48. Vincent JB. Effects of chromium supplementation on body composition, human and animal health, and insulin and glucose metabolism. Curr Opin Clin Nutr Metab Care. 2019;22(6):483-489. doi: 10.1097/MCO.0000000000000604

49. Vincent JB, Lukaski HC. Chromium. Adv Nutr. 2018;9(4):505-506. doi: 10.1093/advances/nmx021

50. VincentJB. New evidence against chromium as an essential trace element. J Nutr. 2017;147(12):2212-2219. doi: 10.3945/jn.117.255901

51. Williams CC, Crochet BT, Bunting LD, Fernandez JM, Stanley CC. Metabolic responses of periparturient holstein cows and heifers supplemented with chromium picolinate. The Professional Animal Scientist. 2004;20(4):312-318. doi: 10.15232/S1080-7446(15)31320-6

52. Zou W, Li C, Hu J, Hou X. Selective determination of $\mathrm{Cr}(\mathrm{VI})$ and non-chromatographic speciation analysis of inorganic chromium by chemical vapor generation-inductively coupled plasma mass spectrometry. Talanta. 2020;218:121128. doi: 10.1016/j.talanta.2020.121128

References

1. Lebedev SV, Gavrish IA, Gubajdullina IZ, Shabunin SV. Effects caused by different doses of dietary chromium nanoparticles fed to broiler chickens. Agricultural Biology. 2019;54(4):820-831. doi: 10.15389/agrobiology.2019.4.820eng

2. Pakholkiv NI, Luchka IV, Dzen EO, Kurtyak BM, Chornushkin BO. Influence of organic and inorganic forms of chromium on cellulolytic and amylolytic activity of microorganisms of rumen CRS. Foothill and Mountain Agriculture and Stockbreeding. 2013;55-1:165-169.

3. Sheida EV, Lebedev SV, Gubaidullina IZ, Ryazanov VA, Gavrish IA. Impact of different forms of chrome on the chemical elements metabolism in the organism of rats of the Wistar line. Izvestia Orenburg State Agrarian University. 2019;2(76):167-171.

4. Lebedev SV, Grechkina VV, Klychkova MV, Mukashev OS. The effect of a mixture of essential amino acids in combination with cobalt and chromium on the chemical composition and quality of beef of the Kazakh white-headed calves. Animal Husbandry and Fodder Production. 2020;103(1):168179. doi: 10.33284/2658-3135-103-1-168

5. Kokorev VA, Mezhevov AB, Gibalkina NI, Fedaev AN, Guryanov AM. Effects of chromium on cow metabolism and dairy productivity. Animal Agriculture and Veterinary Medicine. 2015;3:3-14.

6. Sheida EV, Lebedev SV, Miroshnikov SA, Grechkina VV, Ryazanov VA, Shoshina OV. Changes in the activity of digestive enzymes of pancreatic juice under the influence of ultrafine particles of $\mathrm{Cr} 2 \mathrm{O} 3$ against the background of feeding with protein diets raising cattle. Animal Husbandry and Fodder Production. 2020a;103(4):26-36. doi: 10.33284/2658-3135-103-4-26

7. Kislyakova EM, Lomaeva AA. Influence of organic chrome additives on productive and reproductive indicators of black and pestored breeds. Scientific Notes Kazan Bauman State Academy of Veterinary Medicine. 2017;232(4):76-80. 

$1992 ; 526 \mathrm{p}$.

8. Kononskii AI. Biochemistry of animals. 3rd edition revised and enlarged. Moscow: Kolos;

9. Kravchenko AV. Effect of chromium nanoparticles on quality of carcass, meat and basing fat of pigs. Zootekhnicheskaya nauka Belarusi. 2019;54(2):26-37.

10. Lomaeva AA. Indicators of the productivity of black-moth cows when using organic chromium in diets. [dissertation] Izhevsk; 2018:121 p.

11. Titov VN, Aripovskii AV, Shchekotov VV, Shchekotova AP, Kukharchuk VV. The oleic triglycerides of palm oil and palmitic triglycerides of creamy fat. The reaction of palmitoylation, potassium and magnesium palmitate, absorption of fatty acids by enterocytes and microbiota of large intestine. The Russian Clinical Laboratory Diagnostics Journal. 2016;61(8):452-461. doi: 10.18821/0869-2084-2016-61-8-452-461

12. Sheida EV, Lebedev SV, Miroshnikov SA, Grechkina VV, Ryazanov VA. Assessment of influence of ultrafine particles of $\mathrm{CR}_{2} \mathrm{O}_{3}$ on metabolic processes in the body of calves raised on protein diets. Animal Husbandry and Fodder Production. 2020b;103(4):14-25. doi: 10.33284/2658-3135-103-4-14

13. Kozinets AI, Kozinets TG, Golushko OG, Nadarinskaya MA, Grin MS, Solovyov AV. Primenenie nanochastits khroma $\mathrm{v}$ ratsionakh molodnyaka krupnogo rogatogo skota Application of nanoparticles of chrome in diets of young growth of cattle. (Conference proceedings) Scientific providing livestock production of Siberia: materials IV of the International academic and research conference, (Krasnoyarsk, May 14-15, 2020) compiler. Efimova LV, Lyubimova YuG. Krasnoyarsk: KrasNIIZH FITS KNC SB RAS; 2020a: 257-260.

14. Kokorev VA, Bolotin EV, Rybalkin NI, Fedaev AN, Guryanov AM. Productivity of mature cows at different levels of chromium in their diets. Animal Agriculture and Veterinary Medicine. 2017;2(25):20-30.

15. Kozinets AI, Golushko OG, Kozinets TG, Nadarinskaya MA, Grin MS, Gonakova SA, Soloviev AV. Productivity of calves up to 75 days of age when using chromium nanoparticles Topical Problems of Intensive Development of Livestock. 2020b;23-1:128-135.

16. Skorik NA, Alimov RR. Chromium (III) compounds with some organic ligands. Russian Journal of Inorganic Chemistry. 2020;65(1):16-24. doi: 10.31857/S0044457X2001016X

17. Makaeva AN, Atlanderova KN, Miroshnikov SA, Kosyan DB. Comparative evaluation of feeds digestion effectiveness as result of metal nanoparticles introduction under in vitro conditions. Izvestia Orenburg State Agrarian University. 2017;6(68):178-180.

18. Tuaeva EV, Skovorodnev RV. Effect of feeding chromium mineral and organic form to young cattle in their growth, development and metabolism. (Conference proceedings) Agroindustrial complex: problems and development prospects: materials of the international. scientific-practical conf., dedicated to the Year of Ecology in Russia (Blagoveshchensk, April 5, 2017). Blagoveshchensk: Far East SAU, 2017;1:177-180.

19. Faber W, Akmaliev T, Guseva O. Mineral nutrition for ruminants. Animal Husbandry in Russia. 2020;5:30-33.

20. Sheida EV, Lebedev SV, Gavrish IA, Gubaidullina EZ. Chrome, his role in animal nutrition. (Conference proceedings) Meat cattle breeding - priorities and prospects for development: materials of the International Scientific and Practical Conference (Orenburg, April 25-26, 2018). under the general. RAS Miroshnikov SA. Orenburg: FNC BST RAS; 2018:165-168.

21. Alhidary IA, Alsofi MA, Abdoun KA, Samara EM, Okab AB, Al-Haidary AA. Influence of dietary chromium yeast supplementation on apparent trace elements metabolism in growing camel (Camelus dromedarius) reared under hot summer conditions. Trop Anim Health Prod. 2018;50(3):519524. doi: $10.1007 / \mathrm{s} 11250-017-1462-\mathrm{x}$

22. Baggerman JO, Smith ZK, Thompson AJ, Kim J, Hergenreder JE, Rounds W, Johnson BJ. Chromium propionate supplementation alters animal growth performance, carcass characteristics, and skeletal muscle properties in feedlot steers. Transl Anim Sci. 2020;4(3):txaa146. doi: 10.1093/tas/txaa146

23. Bampidis V, Azimonti G, Bastos ML, Christensen H, Dusemund B, Durjava MK, Kouba M, Lopez-Alonso M, Puente SL, Marcon F, Mayo B, Pechova A, Petkova M, Ramos F, Sanz Y, Villa RE, Woutersen R, Gropp J, Mantovani A, Lopez-Galvez G. Safety and 
efficacy of Availa ${ }^{\circledR} \mathrm{Cr}$ (chromium chelate of DL-methionine) as a feed additive for dairy cows. EFSA J. 2020;18(2):e06026. doi: 10.2903/j.efsa.2020.6026

24. Bompadre TFV, Moretti DB, Sakita GZ, Ieda EH, Martinez MIV, Fernandes EAN, Machado-Neto R, Abdalla AL, Louvandini H. Long-term chromium picolinate supplementation improves colostrum profile of Santa Ines ewe. Biological Trace Element Research. 2020; 193(2):414-421. doi: 10.1007/s12011-019-01741-3

25. Budde AM, Sellins K, Lloyd KE, Wagner JJ, Heldt JS, Spears JW, Engle TE. Effect of zinc source and concentration and chromium supplementation on performance and carcass characteristics in feedlot steers. J Anim Sci. 2019;97(3):1286-1295. doi: 10.1093/jas/skz016

26. Chowdhury S, Pandit K, Roychowdury P, Bhattacharya B. Role of chromium in human metabolism, with special reference to type 2 diabetes. Journal of the Association of Physicians of India. 2003;51:701-705.

27. Deng G, Dyroff SL, Lockart M, Bowman MK, Vincent JB. The effects of the glycation of transferrin on chromium binding and the transport and distribution of chromium in vivo. J Inorg Biochem. 2016;164:26-33. doi: 10.1016/j.jinorgbio.2016.08.008

28. Devoy J, Cosnier F, Bonfanti E, Antoine G, Nunge H, Lambert-Xolin A-M, Decret M-J, Douteau L, Lorcin M, Sebillaud S, Grossmann S, Michaux S, Müller S, Viton S, Seidel C, Gate L. Intraerythrocyte chromium as an indicator of exposure to hexavalent chromium: An in vivo evaluation in intravenous administered rat. Toxicol Lett. 2019;314:133-141. doi: 10.1016/j.toxlet.2019.07.020

29. Edwards KC, KimH, Vincent JB. Release of trivalent chromium from serum transferrin is sufficiently rapid to be physiologically relevant. J Inorg Biochem. 2020;202:110901. doi: 10.1016/j.jinorgbio.2019.110901

30. Feng C, Lin H, Li J, Xie B. Effects of dietary inorganic chromium supplementation on broiler growth performance: a meta-analysis. Peer J. 2021;9: e11097. doi: 10.7717/peerj.11097

31. Haldar S, Samanta S, Banarjee R, Sharma B, Ghosh TK. Glucose tolerance and serum concentrations of hormones and metabolites in goats (Capra hircus) fed diets supplemented with inorganic and organic chromium salts. Animal. 2007;1(3):347-356. doi: 10.1017/S1751731107393026

32. Han M, et al. Effects of organic chromium sources on growth performance, lipid metabolism, antioxidant status, breast amino acid and fatty acid profiles in broilers. J Sci Food Agric. 2021;101(9):3917-3926. doi: 10.1002/jsfa.11053

33. Haq Z, Jain RK, Khan N, Dar MY, Ali S, Gupta M, Varun TK. Recent advances in role of chromium and its antioxidant combinations in poultry nutrition: a review. Veterinary World. 2016;9(12):1392-1399. doi:10.14202/vetworld.2016.1392-1399

34. Hasan HG, Mahmood TJ, Ismael PA. Studies on the relationship between chromium (III) ion and thyroid peroxidase activity in sera of patients with thyroid dysfunction. Ibn AL-Haitham Journal for Pure and Applied Science. 2011;24(2):120-127.

35. Horst EA, Kvidera SK, Mayorga EJ, Shouse CS, Al-Qaisi M, Dickson MJ, Ydstie J, et al. Effect of chromium on bioenergetics and leukocyte dynamics following immunoactivation in lactating Holstein cows. Journal of Dairy Science. 2018;101(6):5515-5530. doi: 10.3168/jds.2017-13899

36. Imamoglu N, Uyanik F, Kocaoğlu GB, Erdem O, Cem LB, Dönmez Altuntaş H. Effects of chromium picolinate on micronucleus frequency and morphology of lymphocytes in calves. Biol Trace Elem Res. 2008;125(2):133-140. doi: 10.1007/s12011-008-8163-2

37. Jobby R, Jha P, Yadav AK, Desai N. Biosorption and biotransformation of hexavalent chromium [Cr(VI)]: A comprehensive review. Chemosphere. 2018;207:255266. doi: 10.1016/j.chemosphere.2018.05.050

38. Kani MM. The effects of different sources of organic and inorganic chromium on blood parameters of broiler chickens. Indian Journal of Science and Technology. 2015;8(28):1 7. doi: $10.17485 / \mathrm{ijst} / 2015 / \mathrm{v} 8 \mathrm{i} 28 / 82778$

39. Lashkari S, Habibian M, Jensen SK. A review on the role of chromium supplementation in ruminant nutrition-effects on productive performance, blood metabolites, antioxidant status, and immunocompetence. Biol Trace Elem Res. 2018;186(2):305-321. doi: 10.1007/s12011-018-1310-5 
40. Lebedev S, Gavrish I, Rusakova E, Kvan O, Gubaidullina I. Influence of various chromium compounds on physiological, morpho-biochemical parameters, and digestive enzymes activity in Wistar rats. Trace Elements and Electrolytes. 2018; 35:242-245.

41. Lu L, Zhao LL, Dong SY, Liao XD, Dong XY, Zhang LY, Luo XG. Dietary supplementation of organic or inorganic chromium modulates the immune responses of broilers vaccinated with Avian Influenza virus vaccine. Animal. 2019;13(5):983-991. doi: 10.1017/S1751731118002379

42. Lukaski HC. Chromium as a supplement. Annu Rev Nutr. 1999;19:279-302. doi: 10.1146/annurev.nutr.19.1.279

43. Spears JW, Lloyd KE, Krafka K. Chromium concentrations in ruminant feed ingredients. J Dairy Sci. 2017;100(5):3584-3590. doi: 10.3168/jds.2016-12153

44. Spears JW. Boron, chromium, manganese, and nickel in agricultural animal production. Biol Trace Elem Res. 2019;188(1):35-44. doi: 10.1007/s12011-018-1529-1

45. Stępniowska A, Tutaj K, Drażbo A, Kozłowski K, Ognik K, Jankowski J. Estimated intestinal absorption of phosphorus and its deposition in chosen tissues, bones and feathers of chickens receiving chromium picolinate or chromium nanoparticles in diet. PLoS One. 2020;15(11):e0242820. doi: 10.1371/journal.pone.0242820

46. Toghyani M, Khodami A, Gheisari AA. Effect of organic and inorganic chromium supplementation on meat quality of heat-stressed broiler chicks. American Journal of Animal and Veterinary Sciences. 2008;3(2):62-67. doi: 10.3844/ajavsp.2008.62.67

47. Untea AE, Varzaru I, Panaite TD, Habeanu M, Ropota M, Olteanu M, Cornescu GM. Effects of chromium supplementation on growth, nutrient digestibility and meat quality of growing pigs. South Afr J Anim Sci. 2017;47(3):332-341. doi: 10.4314/sajas.v47i3.10

48. Vincent JB. Effects of chromium supplementation on body composition, human and animal health, and insulin and glucose metabolism. Curr Opin Clin Nutr Metab Care. 2019;22(6):483-489. doi: 10.1097/MCO.0000000000000604

49. Vincent JB, Lukaski HC. Chromium. Adv Nutr. 2018;9(4):505-506. doi: 10.1093/advances/nmx021

50. VincentJB. New evidence against chromium as an essential trace element. J Nutr. 2017;147(12):2212-2219. doi: 10.3945/jn.117.255901

51. Williams CC, Crochet BT, Bunting LD, Fernandez JM, Stanley CC. Metabolic responses of periparturient holstein cows and heifers supplemented with chromium picolinate. The Professional Animal Scientist. 2004;20(4):312-318. doi: 10.15232/S1080-7446(15)31320-6

52. Zou W, Li C, Hu J, Hou X. Selective determination of Cr(VI) and non-chromatographic speciation analysis of inorganic chromium by chemical vapor generation-inductively coupled plasma mass spectrometry. Talanta. 2020;218:121128. doi: 10.1016/j.talanta.2020.121128

Шошина Оксана Вячеславовна, аспирант 1-го года обучения, Федеральный научный центр биологических систем и агротехнологий Российской академии наук, 460000, г. Оренбург, ул. 9 января д. 29, e-mail: oksana.shoshina.98@mail.ru

Лебедев Святослав Валерьевич, доктор биологических наук, ведущий научный сотрудник лаборатории биологических испытаний и экспертиз, Федеральный научный центр биологических систем и агротехнологий Российской академии наук, 460000, г. Оренбург, ул. 9 Января, 29 , тел.: 8-912-345-87-38, e-mail: 1sv74@1ist.ru

Поберухин Михаил Михайлович, доктор сельскохозяйственных наук, старший научный сотрудник отдела технологии мясного скотоводства и производства говядины, Федеральный научный центр биологических систем и агротехнологий Российской академии наук, 460000, г. Оренбург, ул. 9 Января, 29, тел.: 8(3532)30-81-78

Поступила в редакцию 2 августа 2021 г.; принята после решения редколлегии 13 сентября 2021 г.; опубликована 30 сентября 2021 г. / Received: 2 August 2021; Accepted: 13 September 2021;

Published: 30 September 2021 